\title{
A Simulation module of NS3 for DTN with Time-space model based Routing
}

\author{
Zhihongji Wang ${ }^{1, \mathrm{a}}$, Hao Wu, $\mathrm{W}^{1, \mathrm{~b}}$ \\ ${ }^{1}$ Beijing Institute of Technology, Haidian District, Zhongguancun South Street 5th Beijing, China. The \\ Science and Technology on Information Transmission and Dissemination in Communication Networks \\ Laboratory, the $54^{\text {th }}$ Research Institute of China Electronics \\ a wangzhihongjisina@gmail.com, bwhao@bit.edu.cn
}

Keywords: DTN, Simulation, NS3, Time-space model, Routing.

\begin{abstract}
Under the architecture of Delay-tolerant Networking (DTN), a few routing algorithms and models are introduced to adapt new features different from traditional network. Unlike traditional graph routing algorithm, routings of DTN have the necessity of making use of predicable topology information to overcome the difficult communication challenges, which makes simulation of networking essential. Although the Opportunistic Networking Environment (ONE) Simulator has implemented DTN protocol for simulation, it can't simulate on Linker layer. In this paper, we implement a DTN module based on NS3 network simulator to analyze the performance of time-space model based DTN routing algorithms. Then we provide an analysis of routing experiment using our module with Time-expanded graph (TEG) routing and Contact graph routing (CGR).
\end{abstract}

\section{Introduction}

Space network, consisting of satellites, spacecraft, ground station and planet exploration robot, has the need to overcome many difficult communication challenges [1]: long round trip time (RTTs); the likelihood of data loss due to errors on communication link; possible channel disruptions and etc. Some institutes, specifically, Consultative Committee for Space Data Systems (CCSDS) and Internet Engineering Task Force (IETF) contributes many efforts to create a standard way to solve these problems. As the result of their effort, Delay-and-disruption tolerant networking (DTN) [2] series of protocols are published, intended to become the best solution for communication in space network [3].

The architecture of DTN [4], designed for the Interplanetary Internet, aims to provide Internet like services across interplanetary distances in support of deep space exploration. To achieve that purpose, DTN introduces an overlay protocol, Bundle Protocol that interfaces with both the transport layer and lower layers. With the help of devices implementing the bundle layer, which we called DTN nodes here, the bundle protocol layer can deploy persistent storage to help to conquer network interruptions and delay. While this attribution of space network broken the precondition of the traditional internet protocol which includes continuous connectivity and short delays of one link [1], while routers also perform non-persistent storage and bundle information is persistently stored only at source and destination nodes.

As we stated, the Bundle Protocol (BP) [5] is absolutely the key of DTN, where the basic unit to transfer data between two nearby nodes is a packaged date called bundle, carrying all information into one bundle. The content of this huge bundle includes application layer data and metadata, name of source node and destination node, and any additional data or metadata required for end-to-end delivery, like hop node name and hop time and etc. [6]

What is the most critical in DTN architecture is routing [7], because the routing objective of traditional routing algorithm is an optimal approach to select one path to minimize particular metric, while the routing objective of DTN routing algorithm would consider much more aspects like resource management of small satellites and limit throughput in limit time window [8]. Hence, 
routing in DTN architecture is a complicated topic rather than a path picking problem which has only restricted influence [19].

This paper is organized as follows. In Section 2, we explain the purpose of this paper and the reason why we do it in this way. In Section 3, we first format the routing model and routing process we used in this paper, then we gave the procedure of the simulation. In Section 4, we analyze the result of simulation and get our conclusion of this paper.

\section{Purpose}

As stated in Section 1, routing in DTN needs to be time-space model based instead of traditional topological based. And protocols under linker layer though do not affect performance as much as higher layer, still matter. That's the two original intention of our simulation study. We finally chose to make simulation on NS3 with two time-space model based routing algorithm, with reasons stated below.

There are two main research branches exist in DTN time-space model based routing research area in recent years. One approach is time-expanded graph (TEG) routing, converting space time information to a time-expanded graph $[9,10]$ and apply traditional graph algorithm on them. While another approach is contact graph routing (CGR), accepting a series of node contacts and associated distance ranges in order to form an overall contact graph that identifies the perceived network topology [11] as prior knowledge for each node that can be a basis of routing.

Along with the progress of these two area, many variants and enhanced edition are published by with different aims and different solution [7, 8, 12, 13]. Although these enhanced edition might surmount base edition in some custom environment in some specified target, the comparing between algorithms of different design aims would be meaningless and illogical, which is the reason leads us to choose the base edition of these two algorithm for simulation.

There are many simulator and the ONE simulator is born to work with DTN. We chose NS3 to do so because the ONE can't simulate with linker layer protocols as stated in [14], while simulation with lower layer protocols becomes essential to analyze performance of DTN because the result would be more precise and more detailed.

In Summary, comparing with former work [15]without time-space model based routing, the main contribution of this paper is that we supply a NS3 [16] format module to comparing and analyzing the performance of two time-space model based routing algorithms, as noted before, time-expanded graph routing (or space-time graph) [9] and contact graph routing [17]. To achieve that, this paper implement a DTN software with most fundamental DTN feature like 'carry and delivery', 'neighbor finding', 'retransmission' and etc. With simulation utilities that NS3 supplies, this tiny DTN software can be simulated with custom bundle task plan and custom node moving plan, while source code is available at [18].

\section{Simulation model}

In order to apply different routing algorithm with different network module in this simulation DTN software, we provide different implement and representation of network module and topology for different routing method. In the rest content of this section, we would explain the detail of the routing method and how simulation is made.

\subsection{Time-expanded graph routing}

As noted in Section 2, we choose the algorithm of this paper [9] as a testee. In this chapter, we would make a brief introduce about how we implement it. Considering a wireless network with $n$ moving nodes, with a static set: $V=v 1, v 2, v 3 \ldots$, along with the predicable moving of nodes, we can get the time dynamical representation of this topology as $G(t)=\left(V_{s} E(t)\right)$ where $E(t)$ is a function identifies the link availability through time. The next moving we need to do is transform this $G(t)$ to a time-expanded graph, by 4 steps: 1 . Create a time axis checkpoints sequence, which would separate the whole predicable time into discrete time points. 2. Expand vertex set to time-expanded vertex set 
for each $v n$, giving us a set of vertex as $V t=v 1-0, v 1-1, v 1-2 \ldots v 2-0, v 2-1 \ldots$ by coping static vertex at every time axis checkpoints. 3. Generate a temporal links between time-expanded vertexes that has the same static vertex, and is adjacent in time checkpoints sequence. 4 . Generate a spatial links between time-expanded vertex $v-x-i$ and $v-y-j$ where a link exist between $v-x$ and $v-y$ both in time checkpoints $i$ and $\mathrm{j}$. Note that, this link should have a latency of transmission smaller than time window between checkpoints $\mathrm{i}$ and $\mathrm{j}$, which is presented as edge color set $C=c 1_{s} c 2 \ldots$. Now, we have a time-expanded graph, final step is applying routing algorithm on it to get routing table, which would be used when a routing decision should be made. The pseudo code is available at [9].

\subsection{Contact graph routing}

As noted in Section 2, we choose the algorithm of this paper [19] as a testee and would make a brief introduce about how we implement it in this chapter. With a given wireless graph with moving predicable, we can have the topology presentation as $G(t)=(V, E(t))$. From this $G(t)$, a 'contact plan' can be constructed, descripting contacts between two neighbor nodes consist of contact begin time, contact end time, transmit from node, transmit to node and etc. In CGR algorithm, some parameter needed is unable deduced from $G(t)$, such like node-to-node transmission rate and estimated capacity consumption. For these parameter, we provide an independent way to make assignment. The steps from $G(t)$ to 'contact plan' is as following: 1. Filter all possible con-tact between two nodes in $G(t)$ which distance between nodes is smaller than max transmission range. 2 . For each possible contact, construct a 'xmit' data set with transmission rate and other attribution. 3. Collect 'xmit' of the same 'contact to node' and sort them with the ascending order of the attribution named 'contact begin time'. Now, we have a contact graph, and the final step is applying this CGR to get routing decision. The pseudo code is available at [17].

\subsection{Other routing algorithm}

Except these two routing algorithm, we also provide two naive algorithms as comparison :

1. Spray and wait routing, is a multi-copy scheme routing introduced by article [20], combines the spread speed of multi-copy scheme as first phase and prudent transmission of single-copy scheme as second phase. Spray and wait routing would try to spread one task packet to as much nodes as possible in first phase. While in second phase, Spray and wait would forward packet only to its destination. What worth mentioned is that Spray and wait is the only method applying multi-copy scheme in our simulation.

2. Heuristic routing, is a routing created by us. It would get a topology snapshot of current time, due to the routing decision time. Then it would apply traditional graph shortest path algorithm like Dijsktra on this topology graph snapshot to get the optimal routing decision. This feature might let it make bad decision this time while make a decision against it the next time.

\subsection{Simulation method}

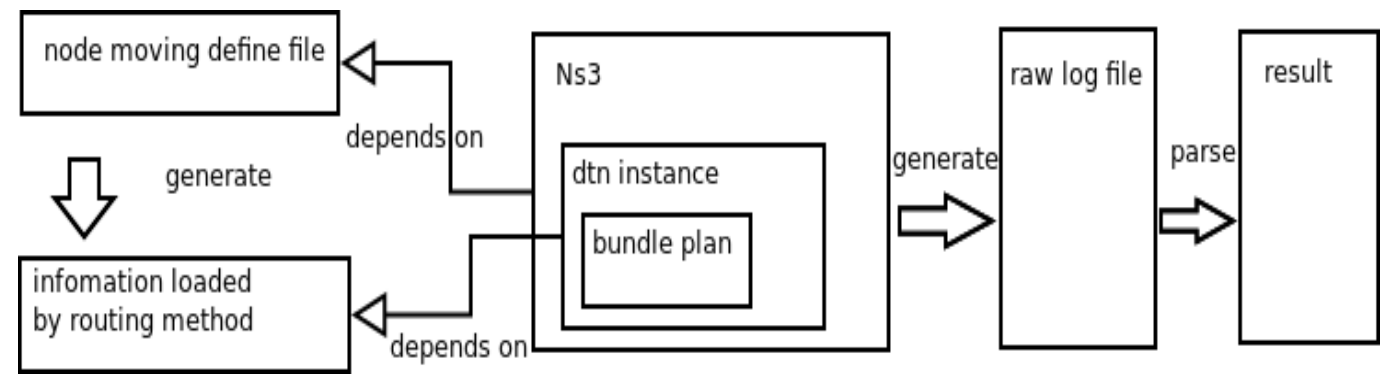

Fig. 1. Procedure of simulation

As noted in the graph fig. 1, this simulation used an outside file to define node-moving in ns3, then abstract node-moving info into a file for DTN instance to generate contact graph and time-expanded graph. After ns3 simulator stopped, the raw output log file would be parsed to get simulation result for us. The detail of protocol stack and network settings can be found in [17]. 


\subsection{Scenario plan}

The bundle plan also matters in this simulation, which is defined as the 'source and sink pair' or 'traffic' of one scenario. Because not all random generated traffic is possible in classical DTN network, we plan it by purpose to keep it as DTN traffic definition says, a disruption possible traffic.

Table 1 Scenario plan

\begin{tabular}{ll}
\hline Scenario name & Bundle amount \\
\hline cycle1 & 4 \\
cycle2 & 16 \\
cycle3 & 36 \\
cycle4 & 59 \\
cycle5 & 92 \\
cycle6 & 146 \\
cycle7 & 217 \\
cycle8 & 336 \\
cycle9 & 504 \\
cycle10 & 672 \\
cycle11 & 1024 \\
cycle12 & 1360 \\
\hline
\end{tabular}

We regard two simulation cases as the same scenario if they have the same node moving plan and bundle plan. The amount of bundles of scenarios used in this paper is descripted as tbl. 1 . What should be mentioned is that these scenarios are not highly relative test case or same test case with different parameter (bundle amount), but different collections of source and sink pairs. That's say, scenario cycle2 may not have a source-sink-pair from node- 10 to node- 4 , while cycle 3 has. The detail of every scenario can be found in the source code of this project.

\subsection{Node moving animation}

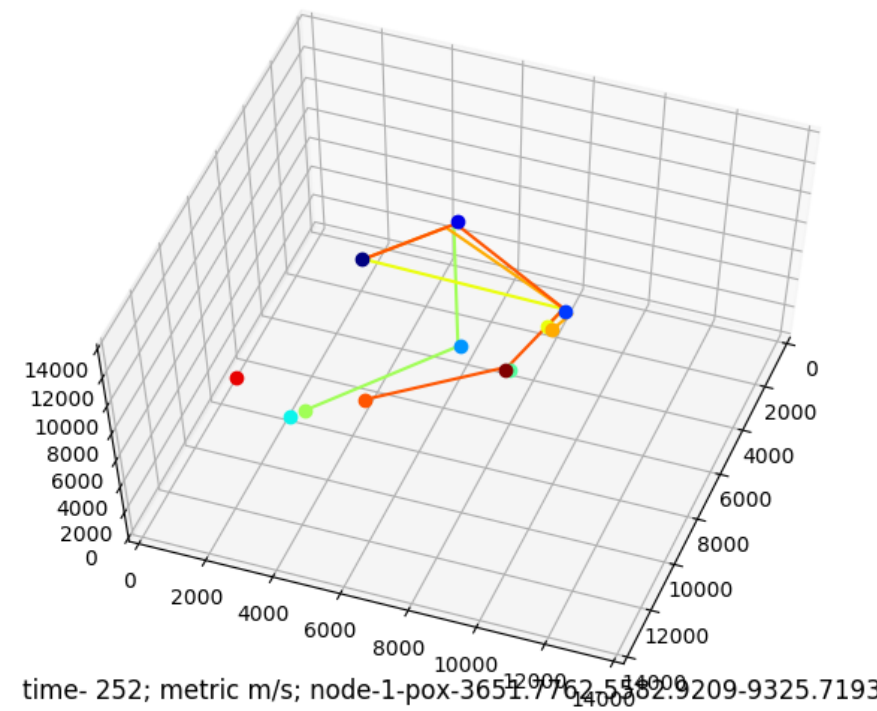

Fig. 2. Nodes moving animation, dynamic topology

The network topology built for analysis is illustrated in following fig. 2, where some nodes almost still during the simulation, while some nodes move around them on clockwise.

With all of these tools we had introduced, we can get our simulation result and make our analysis.

\section{Analysis of simulation result}

\subsection{Result analysis}

Before the analysis of simulation, we should know a fact that the scenarios we have here are formed by different source and sink pairs, as section 3.5 said. This fact would let the curve of result figures seams illogical at first glance, but it's reasonable because they are totally different scenario 
sorted by one metric, bundle amount. The analysis of result would focus on comparison between different routings instead of different scenario.

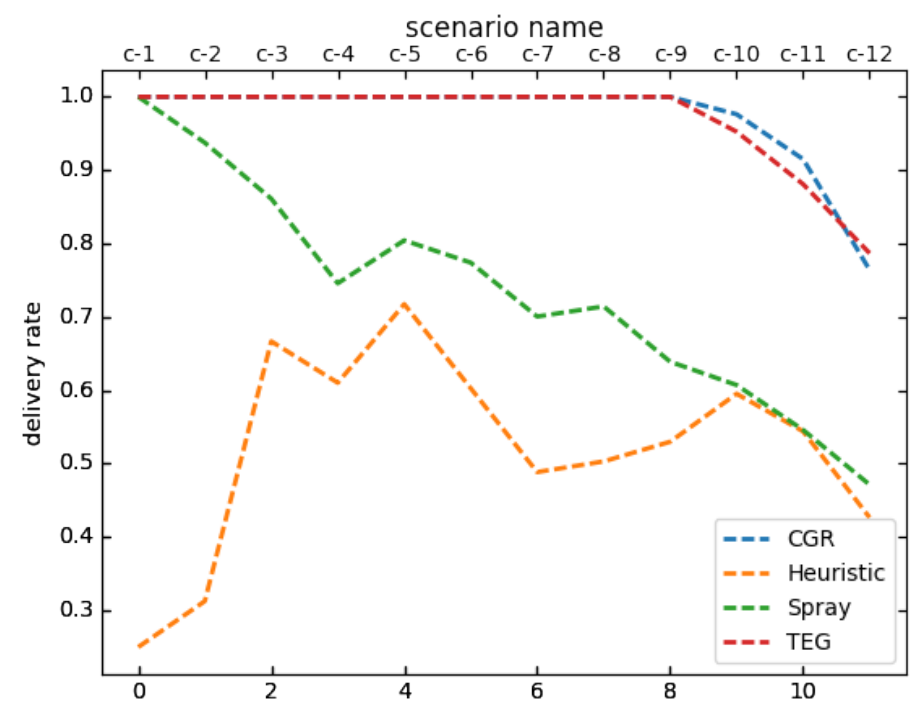

Fig. 3. Delivery rate with different scenario

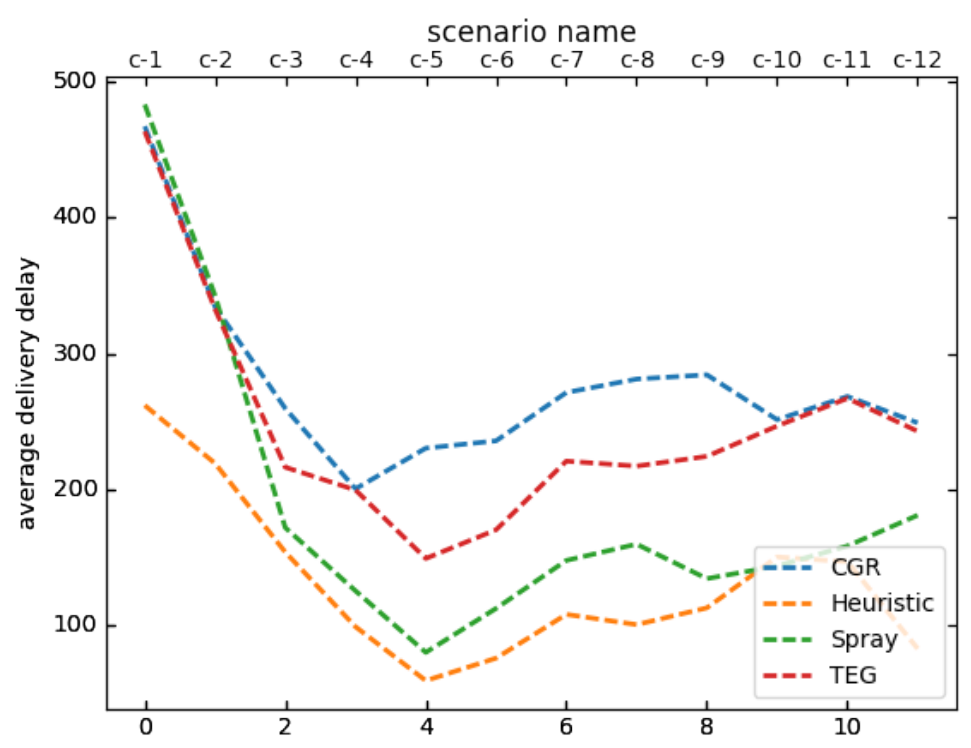

Fig. 4. Delivery delay with different scenario

Fig 3 shows the delivery rate of each scenario, where ' 1.0 ' on y-label means all 'traffic' is achieved and 'c-3' on x-label means that scenario name is 'cycle-3' as tbl. 1 shows. And fig 4 shows the average delivery delay of each scenario, on y-label ' 300 ' means that average delay is 300 s. These three scenarios, 'c-10', 'c-11', 'c-12' reaches the max throughput of this network, and cause the delivery rate of TEG routing and CGR smaller than 100 percentage success. We can tell a few facts from figures:

First, two comparison routings keep low delivery rate while TEG routing and CGR all achieve one hundred percent success until the traffic is bigger than networking available throughput. It matches the assumption we made before that routings in DTN needs to use time-space information to conquer the challenge of disruption.

Second, when traffic is heavy, CGR and TEG performs different delivery rate. Different delivery rates are caused by different path decision along with multiple possible path, under the path decision scheme of their own, CGR with naive scheme vs TEG with best delay scheme. Like most network, each nodes does their routing decision with no direct knowledge of routing of other nodes. So, if two 
nodes choose the same path, name it as Path $-A$, Path $-A$ would arrive its max throughput while Path $-B$, not efficiently used. Although analysis of congestion control is not the purpose of this paper, we can deduce that the effect of algorithm design principle to their performance is remarkable.

Third, as reflected in average delay of fig. 4, the average delay of two comparison routing is better than TEG routing and CGR because the average delay is calculated only by successive deliveries. Due to the fact that comparison routings always have low delivery rate, the performance of TEG routing and CGR are still better than comparison one.

\subsection{Shortcomings of CGR}

As noted in this paper [13], CGR has its own problem, the exhaust search and pick one scheme, of which picking one operation is presented as CGR-Forward Decision affects the routing result. That is to say, in this simulation, we use a naive forward decision with no throughput optimal, which is the factor leads to the result that CGR perform no better than TEG when overhead reach its max throughput. If we can design an advance CGR with best throughput optimal, we can more efficiently use network throughput, which is almost the most cherished performance metric in a DTN network. If we introduce earliest transmission chance into CGR, we can get CGR with Earliest Transmission Opportunity (CGR-ETO) [13].

Another shortcomings of CGR is the lack of buffer management, which would cause non-full utilized of another cherished resource, desk memory of node. If we can introduce more effective buffer management instead of simple expired time procedure, the performance of network with little memory resource would be better.

\subsection{Shortcomings of TEG}

Algorithms in TEG family, which only relies on prior knowledge to make routing, have no resistance to accidental difference of topology. Although, we don't introduces link errors in this paper, we can still say that the bigger the difference is between prior knowledge and realistic of topology, the worse the routing decision is. While the rival of it, CGR has a full concern of difference between prior knowledge and realistic network situation. What is the Achilles' Heel of TEG routing that keep it from real-world usage in DTN software is its unable to utilize dynamic networking information, we think. Still, TEG routing is very easy to implement and handy to make simulation to confirm researcher's new idea, and have its position under the research area.

\subsection{Conclusion}

In this letter, we proposed an ns3 simulation module which can be used to simulate CGR-series and TEG-series routing algorithm, and gave an example of simulation and an analysis of the result. We also discussed the performance of different routing and their cons and pros, which can be deduced from our simulation. The source code of paper is available at [18], and result totally repeatable.

As stated in Section 2, the main contribution of this paper is provide a time-space model based simulation module of NS3. Which can be used to make more precise and detailed simulation result comparing than former work [15].

\section{Acknowledgments}

This work was financially supported by Science and Technology on Communication Networks Laboratory Foundation Project : Aerospace Field Pre-research Foundation Project(060501).

\section{References}

[1] J. van der Geer, J.A.J. Hanraads, R.A. Lupton, The art of writing a scientific article, J. Sci. Commun. 163 (2000) 51-59.

[2] Fall K. A delay-tolerant network architecture for challenged internets. In: Proceedings of the 2003

Conference on Applications, Technologies, Architectures, and Protocols for Computer Communications. ACM; 2003:27-34. 
[3] Caini C, Cruickshank H, Farrell S, Marchese M. Delay-and disruptiontolerant networking (dtn): An alternative solution for future satellite networking applications. Proceedings of the IEEE. 2011;99(11):1980-1997.

[4] Information on: Delay-tolerant networking architecture, internet RFC 4838.

[5] Information on: K. scott and s. burleigh, bundle protocol specifcation, internet RFC 5050.

[6] Information on: Delay-tolerant networking bundle protocol iana registries, internet rfc 6255.

[7] Jain S, Fall K, Patra R. Routing in a Delay Tolerant Network. Vol 34. ACM; 2004.

[8] Xing Y, Ge N, Wang Y. Resource-constrained maximum network throughput on space net-works. Journal of Systems Engineering and Electronics. 2015;26(2):215-223.

[9] Merugu S, Ammar MH, Zegura EW. Routing in Space and Time in Networks with Predicta-ble Mobility. Georgia Institute of Technology; 2004.

[10] Liu R, Sheng M, Lui K-S, Wang X, Wang Y, Zhou D. An analytical framework for resource-limited small satellite networks. IEEE Communications Letters. 2016;20(2):388-391.

[11] Birrane E, Burleigh S, Kasch N. Analysis of the contact graph routing algorithm: Bounding interplanetary paths. Acta Astronautica. 2012;75:108-119.

[12] Li Y, Sheng M, Shi Y, Ma X, Jiao W. Energy efciency and delay tradeoff for time-varying and interference-free wireless networks. IEEE Transactions on Wireless Communications. 2014;13(11):5921-5931.

[13] Bezirgiannidis N, Caini C, Montenero DP, Ruggieri M, Tsaoussidis V. Contact graph routing enhancements for delay tolerant space communications. In: Advanced Satellite Multimedia Sys-tems Conference and the 13th Signal Processing for Space Communications Workshop (Asms/Spsc), 2014 7th. IEEE;

[14] Keränen A, Ott J, Kärkkäinen T. The ONE simulator for DTN protocol evaluation[C]//Proceedings of the 2nd international conference on simulation tools and techniques. ICST (Institute for Computer Sciences, Social-Informatics and Telecommunications Engineering), 2009: 55.

[15] Lakkakorpi J, Ginzboorg P. ns-3 module for routing and congestion control studies in mobile opportunistic dtns[C]//Performance Evaluation of Computer and Telecommunication Systems (SPECTS), 2013 International Symposium on. IEEE, 2013: 46-50.

[16] Information on: https://www.nsnam.org

[17] Caini C, Firrincieli R. Application of contact graph routing to LEO satellite DTN communications[C]//Communications (ICC), 2012 IEEE International Conference on. IEEE, 2012: 3301-3305.

[18] Information on: https://github.com/bit-oh-my-god/ns3-dtnbit.

[19] Information on: https://tools.ietf.org/html/draft-burleigh-dtnrg-cgr-00

[20] Spyropoulos T, Psounis K, Raghavendra CS. Spray and wait: An efcient routing scheme for intermittently connected mobile networks. In: Proceedings of the 2005 Acm Sigcomm Work-shop on Delay-Tolerant Networking. ACM; 2005:252-259. 